\title{
eJRIEPS
}

Ejournal de la recherche sur l'intervention en éducation physique et sport

15 | 2008

Varia

\section{Le sujet en didactique clinique de l'EPS: Conditions et conséquences pour la recherche}

\section{André Terrisse}

\section{(2) OpenEdition}

Journals

Édition électronique

URL : https://journals.openedition.org/ejrieps/5871

DOI : 10.4000/ejrieps.5871

ISSN : 2105-0821

Éditeur

ELLIADD

\section{Référence électronique}

André Terrisse, «Le sujet en didactique clinique de l'EPS: Conditions et conséquences pour la

recherche », eJRIEPS [En ligne], 15 | 2008, mis en ligne le 01 juillet 2008, consulté le 03 mai 2021. URL http://journals.openedition.org/ejrieps/5871 ; DOI : https://doi.org/10.4000/ejrieps.5871

\section{(9) (1)}

La revue eJRIEPS est mise à disposition selon les termes de la Creative Commons Attribution 4.0 International License. 
Le sujet en didactique clinique de l'EPS: Conditions et conséquences pour la recherche

André Térrisse

AP3E du LEMME (EA 3692) et GRIDIFE (ERTe 46), IUFM Midi-Pyrénées,

\section{Résumé}

Dans les recherches sur les pratiques d'enseignement en EPS, la didactique clinique se caractérise par la prise en compte du sujet enseignant. Pour accéder aux origines de son acte, elle croise les concepts de la recherche en didactique et ceux de la psychanalyse, faisant l'hypothèse, maintes fois observée, que l'enseignant décide souvent "à son insu », ce qui constitue une clé à l'intelligibilité des pratiques. Ainsi, seront développés les raisons de cette option scientifique et ses conséquences, notamment celle de valoriser l'analyse au cas par cas et d'utiliser pour ce faire le croisement des concepts de la didactique et ceux de la psychanalyse. Ensuite seront précisés les outils théoriques qu'utilise le chercheur dans sa recherche des causes des événements qu'il observe. Enfin, seront présentés les choix méthodologiques que cette option nécessite, comme la temporalité du recueil de données, le concept d'après coup et l'analyse du rapport de l'enseignant à l'APSA utilisée, dont dépendent les contenus proposés en EPS. L'article se termine en présentant les pistes actuelles de travail de cette équipe de recherche et leurs résultats.

\section{Préambule}

Cette communication du 10 Novembre 2007 aux premières journées Alain Durey, organisées par le GEDIAPS, Université de Paris XII, Val de Marne, s'inscrit dans une continuité. En effet, ce laboratoire m'avait demandé plusieurs interventions :

- le 25 Avril 2001 sur le concept de représentation, depuis longtemps utilisé par Freud (Freud, 1915, cité par Laplanche et Pontalis, 1971), pour l'envisager comme « filtre » du statut des enseignants, en différenciant notamment agent, acteur et sujet.

- le 14 Novembre 2001, sur la question de savoir en quoi les recherches en didactique sont des sciences humaines, question pertinente s'il en est, intervention 
dans laquelle j'avais développé les conditions théoriques et méthodologiques

d'une recherche en didactique de l'EPS (intégrant la dimension du sujet enseignant),

- le 26 Février 2003, sur la question de la subjectivité et sur les problèmes méthodologiques pour les recherches en didactique, où a été présentée la méthodologie clinique développée à l'AP3E du LEMME.

Ainsi, cette communication rassemble les éléments développés dans les précédentes interventions pour en constituer, en quelque sorte, une synthèse. Ainsi seront repris, dans un premier temps, et pour en composer l'introduction, les raisons du choix de la didactique clinique, qui caractérise les travaux que j'ai conduits et ceux que j'ai dirigés.

\section{Introduction : les raisons du choix d'une didactique clinique en EPS}

Trois raisons seront développées :

- la posture du chercheur, que devrait afficher tout chercheur afin de situer clairement ses travaux,

- le projet scientifique, qui spécifie l'orientation d'un universitaire, qui a, non seulement à préciser son champ d'investigation, mais à expliciter les moyens théoriques et méthodologiques de ses études,

- les données recueillies au cours des recherches qui, quelquefois, "déplacent » le chercheur vers d'autres problématiques.

\section{1. La posture clinique}

La posture, qui est à l'origine, " une attitude particulière du corps » (Le Robert, 1985), ce qui convient bien à des études sur l'utilisation des APSA (Activités Physiques, Sportives et Artistiques) en EPS, rend compte du point de vue qu'assume le chercheur dans un champ scientifique, avec ce que ce choix comporte d'options théoriques et de conséquences méthodologiques. Cette posture n'est pas seulement une « approche », un «mouvement par lequel on s'avance » ou une " manière d'aborder un domaine de connaissances quant au point de vue et à la méthode utilisée ", comme l'approche sociologique d'une étude littéraire (Le Robert, déjà cité).

Dans les années 70 a été développée en EPS une « approche clinique », notamment par G Vigarello, qu'il avait précisé dans "Les vertiges de l'intime ", où il prédit " un nouveau stade de l'individualisme...une désaffection du politique et du social au bénéfice d'un rapport nouveau avec la sphère privée » (Vigarello, 1982). Ce texte est certes centré sur le corps, dont il fait le «seul lieu d'aventure ». Mais il marque une orientation qui perdure encore aujourd'hui, celle de la centration sur l'individu, dont il dit que « le retour ne serait 
qu'un total éclairage de soi ». L'orientation dont je me réclame ne se confond pas avec l'hédonisme ou la valorisation du corps. Elle concerne la prise en compte du sujet dans les recherches sur les pratiques enseignantes dont il est :

- l'acteur, qui, au centre de la pièce, joue son rôle, au mieux de ses conceptions et de l'avancée du savoir,

- l'agent de diverses institutions, dans le cadre strict de la discipline scolaire qui est l'EPS, mais aussi dans ses rapports avec l'institution sportive, en rupture ou en adéquation, en fonction de son propre passé de pratiquant ou d'entraîneur,

- le sujet, en tant qu'il est assujetti aux institutions auxquelles il appartient, mais dont quelquefois il se sépare ou qu'il critique. Pour nous, il est surtout le sujet de son inconscient, qui le divise entre des positions quelquefois contradictoires, des sentiments opposés ou des fonctions qu'il cherche à rendre compatibles, comme tenir sa classe et/ou délivrer un savoir.

Cette posture ne se réduit pas non plus à une démarche, de recherche par exemple, qui pourrait alors être coupée de son indispensable support théorique. La posture, de posto, (poser), sous-entend plutôt un positionnement, une «attitude d'esprit », position dans laquelle se tient le chercheur, qui implique non seulement une méthodologie de recherche, mais surtout un choix théorique cohérent avec les objets étudiés. Cette position scientifique a été prise notamment dans mon Habilitation à Diriger des Recherches, soutenue en 1994, dans laquelle apparaît clairement la «prise en compte du sujet » comme objet d'étude des travaux (Terrisse, 1994) sur l'intelligibilité des pratiques d'enseignement, ce qui caractérise les recherches effectuées dans le groupe AP3E (Analyse des Pratiques d'Enseignement et des Effets sur les Elèves) du LEMME.

1. 2. Le projet scientifique

II a pour objet l'observation des pratiques d'enseignement de l'EPS, tout particulièrement lorsque l'enseignant utilise les sports de combat, qui se déclinent en préhension : lutte, judo, et en percussion: boxe anglaise, française, karaté, ..., dans l'enseignement de l'EPS. Ce choix est orienté par ma propre spécialisation, ce qui en constitue une facilité de lectures et d'analyse. Bien entendu, cette observation n'est pas détachée de sa visée de production de recherche qui est de constituer des cadres d'analyse permettant la description et l'explicitation des pratiques d'enseignement de l'EPS.

Si le projet est clair, des difficultés nombreuses, comme dans toute recherche, ne cessent de ralentir le chercheur dans son projet de décrire les pratiques. En effet, il se trouve devant un dilemme : 
- Ou bien les études portent sur un grand nombre d'enseignants pour tenter d'atteindre un certain degré de généralisation. Si cette option entre bien dans les normes scientifiques actuelles de la preuve quantitative, celle qui est valorisée en STAPS notamment, où le chiffre en constitue la garantie, elle interdit les travaux au plus près de l'enseignant, que l'on pourrait caractériser de «micro didactiques ".

- Ou bien, et c'est cette deuxième option qui a été prise dans nos travaux, l'observation se centre sur un enseignant singulier, qui permet une analyse approfondie de son activité que ne permet pas la première option, au point d'envisager de produire, avec son concours, les «raisons" de son acte, orientation que je désigne sous le nom de "didactique clinique ».

Ce projet se heurte non seulement à des difficultés de description, dans l'opposition singularité-généralisation, mais aussi à son objet même : la contingence de toute situation d'enseignement (Brousseau, 1998). Brousseau depuis longtemps a montré combien la transmission d'un savoir ne va pas de soi et combien le professeur est confronté à un " indécidable », puisque l'appropriation du savoir est sous la responsabilité de l'élève (Terrisse, déjà cité). Cette catégorie logique rend compte du fait que la situation didactique n'est jamais maîtrisable et que le professeur ne sait pas quand l'élève apprend, ce qu'il apprend et pourquoi il l'apprend. En se référant à $M$ Verret, tout professeur sait « que tout le savoir ne peut être transmis ", ce que M Verret nomme l'indéductible car il ne sait «ce que les enseignés feront de ce que l'on leur aura enseigné » (Verret, cité par Terrisse, Léziart, 1997).

\section{3. La réalité de l'observation.}

D'autre part, la réalité de l'observation met en évidence deux questions : la singularité des pratiques et l'énigme de certains actes.

La singularité des pratiques constitue un fait incontournable qu'observe tout chercheur dès lors qu'il s'intéresse aux pratiques d'enseignement in situ. Dès les premières recherches menées dans le groupe AP3E, un chercheur a observé, malgré une négociation « serrée " du script didactique avec les enseignants concernés, des transformations du savoir enseigné importantes, qui fait dire au chercheur que « les contenus (d'enseignement) ne sont jamais stabilisés " (Carnus, 2001). La recherche en didactique doit prendre acte de ce processus, somme toute tout à fait normal d'une labilité des contenus soumis aux aléas de la classe, et en tirer les conséquences qui s'imposent. Le rapport personnel qu'entretient tel professeur avec tel savoir, sa position institutionnelle (stagiaire ou « expérimenté »), la façon de considérer sa fonction de professeur, du fait qu'il est « sujet 
supposé savoir » (Lacan, cité par Chevallard, 1985), vont largement déterminer à la fois sa conception du savoir et surtout la façon dont il va le transmettre.

Deuxième observation: l'énigme de certains actes. Comme l'a écrit très justement Perrenoud, le professeur « décide dans l'urgence » face à l'incertitude que constitue la transmission même d'un savoir (Perrenoud, 1996). Nous pouvons observer que l'enseignant est effectivement traversé, comme tout sujet, de tendances quelques fois contradictoires, de dilemmes (Ria, 2001), qui le renvoient à ses propres divisions. Devant l'obligation de décider, même si la non-décision peut être considérée comme une décision, il fait des choix de situations didactiques, de modifications de variables, de critères d'évaluation...qui quelquefois interrogent le chercheur dans la compréhension qu'il tente d'avoir de ces pratiques. Notre posture clinique nous incite à penser qu'il y a bien une raison que le chercheur doit extraire et qui a un rapport avec l'apprentissage des élèves. Comme l'écrit Lévy-Strauss, il y a toujours une raison au fait humain observé, et c'est au chercheur à la produire (Lévy-Strauss, 1962).

Les raisons du choix de la didactique clinique ayant été précisées, voyons quelles en sont les références théoriques.

\section{Cadre théorique : le croisement de deux champs scientifiques.}

2. 1. La recherche au « cas par cas»

Comme nous l'avons déjà écrit, la clinique se définit d'abord (mais pas seulement), par « le cas par cas », soit la prise en compte incontournable de la singularité du sujet, pris un par un. Ce point de vue valorise les études de cas, puisque le terrain d'observation est toujours unique. Ce choix implique des conséquences sur le traitement de ces données très «contextualisées », en rapport avec le cadre théorique. Notamment, il pose la question de la validité scientifique de telles études, confrontées à un environnement très quantitatif des recherches en éducation, même si la tendance s'inverse actuellement. Ce choix exige une méthodologie de «construction du cas », déjà développée en psychanalyse par Freud, qui a réussi à faire passer le cas personnel à l'universel de tout humain, lors qu'il décrit par exemple le complexe de castration, auquel tout sujet est inscrit, mais différemment pour chacun d'eux (Freud, 1985).

Dans de telles recherches, l'étude de cas permet de prendre en compte l'incontournable singularité des cas observés et de tenter d'accéder aux raisons de leurs actes, soit de pouvoir répondre à la question du pourquoi : pourquoi cet enseignant change $t$ il de milieu alors que la situation didactique ne l'exige pas, ou pourquoi cet enseignant introduit il un nouveau savoir qu'il n'avait pas prévu ? Elles tentent, dans leurs visées, de réintroduire la 
question de la cause, à partir du moment où une réponse personnelle intervient dans l'enseignement, en rapport très souvent avec le passé « sportif » ou professionnel de l'enseignant.

Cette remarque met en évidence la double détermination d'un fait didactique. En effet, il est déterminé par des contraintes :

- Didactiques, comme l'établissement scolaire, le niveau des élèves et la nature même du savoir enseigné,

- «Internes » à l'enseignant qui le renvoie à sa propre histoire d'enseignant, sa formation, sa pratique de l'activité enseignée, voire aux signifiants familiaux qui l'ont conduit à choisir ce métier.

2. 2. Les deux champs de référence.

Si la didactique clinique se caractérise par le croisement de deux ordres de détermination dans l'explication d'un fait didactique, s'impose alors la référence à deux champs théoriques.

\section{2. 1. Les concepts de la didactique}

Ce champ scientifique concerne l'utilisation des trois concepts clés: la transposition didactique, le contrat didactique et le rapport au savoir (même si ce dernier renvoie à plusieurs définitions), (Terrisse, 2005), outils indispensables à rendre compte du devenir du savoir au cours des différentes phases de son enseignement.

L'expérience de recherche nous incite à privilégier dans le cadre restreint de cet article deux types d'analyse.

D'une part l'articulation dévolution/institutionnalisation. En référence à la théorie des situations didactiques de G Brousseau, cette articulation renvoie à deux moments clés de l'activité de l'enseignant : celle où il dévolue à l'élève la responsabilité de l'apprentissage, dont l'issue, comme dans le sport, est toujours contingente (l'apprentissage peut se produire ou ne pas se produire) et celle où il rend public, à l'ensemble des élèves, le savoir visé, qui fixe de manière symbolique la dernière phase de son enseignement et l'atteinte du savoir. Cette relation contingent-nécessaire ...ancrage symbolique nous semble être un cadre d'analyse pertinent du processus d'enseignement-apprentissage.

D'autre part le processus d'ostension. D'autres auteurs ont déjà travaillé sur cette question de la monstration (Salin, 2002). Elle est aussi pertinente dans la mesure où le « savoir doit s'avancer sous le masque »(Brousseau, déjà cité), mais le professeur doit mettre en place les conditions de sa découverte par l'élève, ce qui le conduit quelquefois à des aides plus directes. En EPS, la démonstration a pour but de montrer le geste à réaliser et s'oppose ainsi au processus de la dévolution. Toutefois, il revient à l'élève de le réaliser, 
ce qui n'est pas toujours facile, et de l'utiliser souvent dans des conditions bien précises, qui lui donnent un statut de savoir efficace. Nous pensons ici aux «prises » de lutte ou de judo, qu'il est quelquefois difficile de réaliser en soi (en coopération), mais qu'il est encore plus difficile d'utiliser dans un combat pour déséquilibrer son adversaire (en opposition), ce qui est sa fonction. L'ostension est intéressante à étudier, car plusieurs formes se combinent, de la plus «directe » à la plus «déguisée », en fonction du but recherché et de la stratégie de l'enseignant. Ce moyen, qui s'apparente à un « geste professionnel », nous paraît rendre compte d'une articulation chère à G Brousseau : l'articulation possible-nécessaire. En effet, le professeur a le choix de plusieurs modes d'ostension pour faciliter l'apprentissage, jusqu'à trouver celle qui deviendra nécessaire à l'apprentissage. Elle devient alors, pour le chercheur, un indice de sa professionnalité, peut être même de sa compétence.

2. 2. 2. Les concepts de la clinique psychanalytique

Dans le cadre de cette présentation, nous en retiendrons deux :

- La division du sujet, qui s'écrit $S$ barré, $\$$, et qui rend compte du fait que l'enseignant, comme tout sujet, est traversé de plusieurs divisions. L'une d'entre elles nous paraît évidente à mettre en valeur, c'est celle qui correspond à la différence entre un savoir idéal qu'il voudrait transmettre et le niveau réel des élèves qui ne lui permet pas toujours de conserver cet idéal. Freud a déjà écrit qu'enseigner était « un métier impossible » (Freud, 1973). Une troisième articulation (qui s'ajoute aux deux qui viennent d'être présentées) pourrait alors devenir un cadre d'analyse du chercheur, c'est le rapport idéal-réel. L'observation serait alors sensible à la façon dont l'enseignant « négocie » son intention didactique en fonction des difficultés qu'il rencontre «sur le terrain », comme la résistance des élèves à entrer dans son univers de valeurs, de normes et de savoirs.

- Le « sujet supposé savoir», expression empruntée par Y Chevallard à Lacan. Cette expression renvoie, d'après nous, à la place symbolique qu'occupe, qu'il le veuille ou non, l'enseignant. Cette expression nous paraît particulièrement pertinente lorsqu'on étudie les pratiques d'enseignants débutants, car l'observation nous montre qu'ils sont souvent à la recherche d'une "légitimité didactique », sachant très bien combien il leur sera difficile de se faire reconnaître par les élèves, qui savent bien qu'ils sont stagiaires (Buznic, 2005). Elle est aussi très utile dans l'observation d'enseignants « expérimentés » (qui ont plusieurs années de pratique professionnelle) mais qui sont déstabilisés par l'enseignement d'une APSA qu'ils ne connaissent pas (Terrisse, Lestel, 2005). Si leur légitimité didactique n'est pas 
entamée, dans la mesure où ils « maîtrisent » leur enseignement, des difficultés apparaissent dans le traitement de l'activité qui fragilise leur statut même. Dans une recherche menée dans l'AP3E, une enseignante se plaignait de «manquer de temps », alors que cette plainte récurrente masquait une difficulté de prévoir un contenu d'enseignement pertinent : « Je manquais de temps au lieu de te dire que c'est le contenu qu'il me manquait », (Terrisse, Lestel, déjà cité), aveu qui justifie la centration de recherche sur l'articulation savoir-sujet, objet central d'étude de la didactique clinique.

Pour terminer la présentation de ce cadre, nous pourrions caractériser la didactique clinique par deux points :

- Son objet est bien de rendre compte du « devenir» du savoir, depuis la référence qu'a choisie l'enseignant, aux multiples transformations qu'il lui fait subir du fait des « résistances» des élèves, voire des conditions mêmes d'exercice du professeur.

- Sa visée est de tenter d'extraire les « causes » de ces transformations, qui tiennent à l'histoire personnelle de l'enseignant, et de son rapport à l'APSA qu'il utilise dans ses cours en EPS.

Au croisement de ces deux champs scientifiques, quels sont alors les objets d'étude privilégiés en didactique clinique?

\section{Les objets analysés en didactique clinique de l'EPS}

3. 1. La question du savoir dans la didactique des APS (Terrisse, 1994) Les références théoriques qui orientent actuellement mes travaux personnels et ceux que je dirige se trouvent dans mon Habilitation à Diriger des Recherches, en 1994. Dans la note de synthèse, je développe en effet le cadre d'analyse qui sert ici de référence. J'utilisais alors le « triangle didactique », en précisant plusieurs points :

1. le savoir n'est pas seulement conçu comme le troisième terme du triangle, il est envisagé au centre du triangle et se décline au pluriel, comme l'ont montré plusieurs auteurs (Van Der Maren, 1996, Caillot, 2001).

2. J'assimile l'enseignant à un sportif, qui ne connaît pas par avance le résultat à l'épreuve sportive. La transmission du savoir est bien une épreuve au sens où l'issue n'est pas prévisible et où l'enseignant éprouve son savoir à la réalité de la classe.

3. L'articulation des trois côtés est soumise aux catégories logiques : 
- Du nécessaire, puisqu'il est nécessaire que le savoir soit acquis et défini par l'enseignant pour être transmis,

- Du possible, ou des possibles que l'enseignant a comme choix dans son enseignement,

- Et de l'indécidable, qui rend compte du fait que cette transmission n'est jamais assurée.

3. 2. La catégorie de l'impossible (Terrisse, 2002)

Au fil des travaux et des directions de thèses, apparaît une nouvelle catégorie qui rend mieux compte de la difficulté à enseigner, celle de l'impossible. Sans reprendre l'article : "au-delà du didactique, le clinique " (Terrisse, 2002), je faisais alors valoir que l'enseignant se heurte à deux difficultés, inhérentes à la transmission du savoir :

- il ne peut pas tout transmettre aux élèves, du fait de la complexité et de la multitude des savoirs contenus dans une situation ou dans une APSA. E Margnes montre que le professeur de judo enseigne au moins cinq savoirs différents: technique, stratégique, sécuritaire, éthique et réglementaires (Margnes, 2002).

- II est confronté au réel, soit ce qui échappe, par définition, à pouvoir être dit, qui ne passe pas à la symbolisation, que Lacan définit comme «l'impossible à dire ", ou encore comme " ce qui ne cesse pas de ne pas s'écrire » (Chémama, 1995), ce que l'on retrouve en EPS sous la forme de sensations corporelles, d'affects, de perceptions difficiles ou impossibles à dire.

Tableau I. La catégorie de l'impossible, Impulsions (Terrisse, 2002).

\begin{tabular}{|c|c|}
\hline Nécessaire & $\begin{array}{c}\text { Le réel, } \\
\text { Impossible à dire }\end{array}$ \\
\hline SAVOIR Y FAIRE & Indécidable \\
\hline Possible (s) & $\longrightarrow \quad$ Impossible \\
& (pas tout le savoir se transmet) \\
\hline
\end{tabular}


3. 3. Un enseignant assujetti

L'enseignant, comme tout sujet, est par définition, assujetti, notamment à plusieurs institutions. La première est celle de l'institution scolaire qui pèse lourdement sur l'enseignement de cette discipline, du fait de son statut à part à l'école, démontré par les historiens de l'EPS (During, 1981). Enseigner une séance de sport collectif en EPS se différencie nettement de ce qui peut être enseigné en club...bien que cette différence soit moins évidente en judo, dont l'enseignement est très proche de ce qui se fait en club, du fait de la formation fédérale des professeurs du judo (Loizon, 2004).

L'institution sportive influence l'enseignement de l'EPS, influence que les didacticiens ont trop longtemps sous-estimée, plus par idéologie que par observation rigoureuse. Dans ce cadre, on se doit de mentionner des différences essentielles entre les institutions de formation des années 1960-1970, entre les CREPS (Jeunesse et Sports) et les UEREPS (Faculté de médecine), ou actuellement entre les UFRSTAPS (Université) et les IUFM (Instituts de Formation des Maîtres).

Enfin, plusieurs autres institutions ont une influence sur les pratiques d'enseignement, comme l'inscription familiale, l'origine sociale, l'appartenance professionnelle qui sécrète (et quelquefois impose) une "culture professionnelle", les engagements politiques, ethniques ou religieux.

Ces différents assujettissements pèsent sur l'enseignement d'une discipline scolaire comme l'EPS que le chercheur a pour fonction d'identifier, quand il le peut.

3. 4. Un enseignant singulier

Nos recherches montrent que toute observation des pratiques des enseignants repose chaque fois sur un "inédit», une forme nouvelle et chaque fois renouvelée de la transmission d'un savoir. Récemment, dans les travaux du GRIDIFE (ERTe 46 de l'IUFM Midi-Pyrénées), les chercheurs en physique-chimie ont proposé le même script didactique à plusieurs professeurs stagiaires. Ils ont observé autant de pratiques que d'enseignants, alors que le contenu avait été préparé par l'ensemble des stagiaires (Calmettes, SaintGeorges, Flandé, à paraître). Même si le contenu d'enseignement a été défini, chaque professeur y apporte sa touche personnelle et modifie ainsi le savoir à enseigner luimême, ce qui paraît être une des conditions de sa transmission.

Chaque enseignant est confronté à un impossible à enseigner, qui se décline différemment selon chacun. Toutefois, pour y parvenir, tous développent un "savoir y faire » avec la contingence de toute situation didactique, mais où le « $y$ » introduit la marque de la singularité. 
3. 5. Un enseignant divisé

Plusieurs travaux ont montré combien l'enseignant, comme tout sujet, est divisé entre :

- Ce qu'il prévoit de faire et ce qu'il peut faire, dans le contexte toujours mouvant de la classe (Carnus, 2001).

- Ce qu'il fait en classe et ce qu'il peut en dire après la classe. Loizon a montré que les professeurs de judo mobilisent plus de variables didactiques qu'ils n'en peuvent nommer (Loizon, 2004).

- Ce qu'il dit de ce qu'il a fait et ce qu'il ne peut s'empêcher de faire, du fait de son histoire de pratiquant ou d'enseignant (Buznic, 2005 ; Terrisse, Lestel, 2005).

Ces divisions deviennent un cadre d'analyse très pertinent et peuvent être utilisées pour tout enseignant, divisé entre le «programme » imposé et le temps nécessaire pour y parvenir.

3. 6. Un enseignant désirant

Cette option scientifique n'oublie pas la référence à $\mathrm{J}$ Beillerot, pour qui le rapport au savoir ne peut s'étudier sans soulever la question du désir de savoir (Beillerot, 1996). Non seulement l'apprentissage repose sur un désir, celui de plaire à ses parents, à ses copains ou éventuellement à son professeur, mais aussi peut être sur celui de régler un problème de relation personnel à un autre élève (Sauvegrain, 2001). Plusieurs recherches montrent en effet l'importance pour les enseignants de vouloir transmettre « le plaisir » de la danse ou la «stratégie » du combat en judo, ce que l'enseignant lui-même a trouvé comme intérêt dans sa propre pratique.

En dehors du désir de répondre, plus ou moins, à la demande institutionnelle, chacun a à cœur de transmettre quelque chose de lui, difficile d'ailleurs à extraire et à dire, un savoir personnel et souvent inédit. Le désir soutient leur enseignement.

A contrario, il est possible d'accéder à l'importance de ce désir lorsqu'il est défaillant, par exemple dans l'enseignement d'une activité non pratiquée par l'enseignant.

Ayant précisé les objets étudiés dans cette option, développons maintenant les outils méthodologiques utilisés.

\section{Les options méthodologiques}

4. 1. La temporalité

Afin de suivre l'enseignant pas à pas, au fil de son enseignement, nous utilisons une méthodologie temporelle de recueils de données, dont l'après coup est une des dernières étapes, ayant pour fonction, non seulement de revenir sur le déroulement des séances 
d'EPS, mais aussi de produire une reformulation de ce qui s'est passé, quelquefois même un sens nouveau, selon le tableau suivant :

Tableau II. Le recueil temporel des données.

\begin{tabular}{|c|c|c|}
\hline Déjà là & Épreuve & Après coup \\
\hline $\begin{array}{c}\text { Conception de l'APSA } \\
\text { Conception de son } \\
\text { enseignement }\end{array}$ & Observation in situ : & Retour sur les séances \\
confrontation du savoir à & d'EPS : nouvelle \\
la classe & formulation \\
\hline
\end{tabular}

\section{2. Le concept d'après coup}

Ce concept a été emprunté à la psychanalyse et cité par Chevallard (Chevallard, 1985) pour rendre compte du principe de « remaniement ». En effet, en psychanalyse, le savoir ne se sait qu'après coup puisque « des impressions ou des traces mnésiques peuvent n'acquérir tout leur sens, toute leur efficacité que dans un temps postérieur à celui de leur première inscription »(Chémama, 1995). La recherche en didactique clinique de l'EPS doit mettre en place une méthodologie permettant à ce « refoulé » professionnel d'être pris en considération dans la tentative d'explication des faits et gestes des enseignants, notamment quand le chercheur n'en a pas la clé, ce qui nous paraît être le sens de la recherche en didactique. Ce concept devient alors une des conditions pour que le savoir s'écrive.

La vérité d'un acte d'enseignement ne se déduit pas spontanément de son observation. Elle n'est quelquefois pas consciente par l'enseignant lui-même. Elle a besoin à la fois d'un outil théorique, qui pose les exigences d'un retour sur soi, d'un passage par la parole, et des conditions méthodologiques d'une production de sens certes déjà présent dans l'acte, mais non encore élucidé. Ce processus repose sur la responsabilité du sujet « dans ce qui lui arrive » à travers les « dires », «l'insu », « ce qui ne marche pas » (Terrisse, 2000).

Ainsi, le chercheur est plus sensible aux hésitations, aux lapsus, aux contradictions qui caractérisent la recherche de la vérité du sujet qu'aux déclarations qui vont de soi.

\section{3. Le rapport à I'APSA}

La conduite de nos travaux nous montre combien le rapport qu'entretient le professeur d'EPS à l'APSA enseignée détermine grandement la façon qu'il a de la transmettre à partir de la conception qu'il en a. Ce pourrait être une banalité de penser que ce rapport induit 
son enseignement. En fait, à partir du moment où le chercheur tente de décrire et de suivre pas à pas le savoir enseigné, cette donnée devient essentielle, car l'enseignant d'EPS ne transmet pas seulement un savoir désincarné, vidé de sa substance corporelle. Il transmet le plus souvent une expérience personnelle, faite de sentiments, d'affects, de peurs, de plaisir, difficiles à exprimer, si ce n'est sous la forme de métaphores ou d'expressions qui lui sont propres. Ainsi, il peut transmettre une passion, la danse : « je me suis régalée » ou, au contraire une aversion pour la lutte: «j'ai détesté...me battre, moi, c'est pas trop ça » (Buznic, 2005).

Ce qui est recherché n'est pas le rapport à l'APSA en lui-même, mais ce que ce rapport produit comme effet sur l'enseignement. Par exemple, cette professeur stagiaire d'EPS trouve dans son activité préférée un moyen de reconnaissance auprès des élèves : "c'est vrai, la démonstration, c'est aussi leur en mettre plein la vue ", alors que la lutte, qu'elle enseigne la même semaine, avec les mêmes élèves la limite nettement : " tu ne montres pas parce que tu es flag (pris en flagrant délit), donc tu te discrédites ".

Ainsi, une problématique de recherche peut s'élaborer autour de la comparaison, pour le même enseignant, avec les mêmes élèves et dans une même semaine, d'un enseignement " contrasté » de deux APSA pour lesquelles l'enseignant n'a pas le même rapport de pratique personnelle.

4. 4. Le rapport au corps (Brousse, Labridy, 1983)

Dans ces recherches sur l'enseignement de l'EPS à des fins de production d'outils d'analyse de ces pratiques, le statut si particulier du corps est oublié, au moment même où le corps devient, comme dans les années 1970, une préoccupation de recherche (Andrieux, 2007).

$\mathrm{M} \mathrm{H}$ Brousse et $\mathrm{F}$ Labridy, dans un texte déjà ancien de la revue EPS, posaient clairement le statut multiple du corps, en référence au texte de Lacan sur RSI (Lacan, 1975). En effet, le corps, dans cette référence est à la fois :

- Une image, à laquelle sont sensibles les professeurs d'EPS, image qui peut devenir un moyen de capter l'attention des élèves, leur admiration. Mais cette image, malheureusement peut se dégrader : le corps n'est pas seulement « glorieux ».

- Un signifiant, qui soutient l'enseignant dans sa pratique quotidienne, et notamment son statut, car il est le «sujet supposé savoir ». Cette fonction est toujours difficile à assumer, puisque dans leur article, M H Brousse et F Labridy leur conseillent « d'avoir le courage de (leur) propre bêtise », soit de ne pas vouloir s'équivaloir à cette fonction.

- Un objet, puisque le corps se matérialise par une forme visible, un ensemble de muscles, dos, d'articulations qui permettent l'apprentissage moteur et la performance 
sportive. Mais sa fragilité se manifeste pour des sujets qui en font leur faire valoir ou "leur être au monde». En effet, le sportif est atteint, comme tout sujet, par les défaillances du corps, comme les accidents, les contre-performances ou les abandons.

\section{Résultats à partir de travaux actuels.}

Même si le contexte de recherche change, au gré du changement de statut des laboratoires, plusieurs résultats confortent les pistes actuellement explorées en didactique clinique :

5. 1. La différence débutants-expérimentés.

Ce thème constituait l'axe de recherche de l'ERTe 46 (GRIDIFE) de 2002 à 2006. L'objectif de cette production scientifique est d'élaborer des connaissances sur le fonctionnement des enseignants débutants en décrivant au mieux les difficultés qu'ils rencontrent. Ces connaissances s'avèrent utiles pour construire et conduire les modules de formation initiale, mais aussi pour les chercheurs travaillant sur les pratiques d'enseignement, car le débutant actualise et grossit les difficultés inhérentes à toute transmission de savoir. Dans les travaux initiés dans le groupe AP3E, deux éléments ont retenu notre attention :

- l'avancée du temps didactique. En comparant deux enseignants, l'un débutant, l'autre expérimenté, nous nous sommes rendus compte d'un rapport au temps différent. Le débutant avance "en même temps que les élèves » alors que nous avons observé chez l'expérimenté une anticipation du temps didactique par l'observation, par l'aménagement du milieu et par l'utilisation de régulations justifiées.

- La position symbolique de l'enseignant. Du fait d'une position statutaire mal assurée, nous avons observé qu'un professeur stagiaire recherchait avidement une "légitimité didactique » à laquelle l'expérimenté n'est pas aussi soumis. De ce fait, un certain nombre de gestes, de paroles nous semble non pas orientés vers le savoir à transmettre, mais vers une position symbolique à assurer.

5. 2. L'analyse différentielle d'un même enseignant.

Nous utilisons la comparaison de deux enseignements, pour un même enseignant, l'un concerne une APSA qu'il a déjà enseignée, l'autre est une activité qu'il connaît peu ou qu'il n'a pas encore enseignée.

Nous pouvons alors analyser sa pratique à travers l'outil ressemblances-différences. Les ressemblances renvoient à ses caractéristiques propres, son style ou encore sa signature 
(Blanchard-Laville, 2001). Chez un même enseignant, apparaissent des éléments qui le spécifient, comme :

- La primauté de la technique.

- Le "manque de temps », qui apparaît comme une plainte récurrente, quelle que soit l'activité enseignée.

- L'ostension verbale signe sa façon de s'adresser aux élèves de cette classe.

- Un désir de «maîtriser » cette classe, ou comme l'écrit le chercheur, de «forclore l'incertitude ».

Par contre l'observateur remarque des différences importantes, comme :

- Une conception et un traitement différents des deux activités, alors qu'elles sont toutes les deux des activités d'opposition.

- Un rapport différent au temps didactique, d'anticipation pour l'une, de « crise temporelle » pour l'autre.

5. 3. Le poids de l'expérience antérieure dans une APSA.

Dans cette option, l'objectif est de mesurer le poids de l'expertise corporelle sur l'enseignement de l'EPS. L'expertise correspond à une connaissance prouvée depuis longtemps, quelquefois dès l'enfance, d'une spécialité sportive sous la forme de pratique de haut niveau, ayant même servi de choix d'option au CAPEPS quelquefois.

L'enseignement de sa " spécialité » permet d'observer chez l'enseignant :

- Le respect de la logique interne de l'activité, intégrée depuis longtemps par l'enseignant.

- L'identification des niveaux des élèves facilitée par la connaissance «intime » des transformations motrices induites par la pratique physique.

- La qualité des régulations, dès lors que l'enseignant sait ce que l'apprentissage moteur permet d'obtenir.

Par contre, dans une activité méconnue, nous observons chez ce même enseignant :

- Une difficulté récurrente à comprendre les difficultés d'apprentissage des élèves, comme les formes de corps efficaces ou les enchaînements moteurs nécessaires à la réalisation d'un mouvement.

- Une constante hésitation sur le traitement didactique de l'activité, revenant souvent sur sa feuille de préparation, gage du projet d'enseignement.

Pour cette activité, l'enseignante est consciente de ses difficultés qu'elle identifie dans cette formule : «il me manquait de sentir l'activité ». 
Ces travaux demandent à être poursuivis pour affiner ces cadres d'analyse. C'est ce que les prochaines thèses fourniront comme savoirs.

\section{Discussion}

Dans la mesure où cette contribution a pour objet de présenter une orientation scientifique en didactique clinique, dans le cadre des recherches sur l'intervention de l'enseignant d'EPS, nous avons volontairement réduit les concepts utilisés en didactique et en psychanalyse pour qu'elle puisse entrer dans le cadre de cette publication.

D'autre part, le lecteur a du se rendre compte que nous sommes restés dans la stricte référence à « l'inventeur » de la psychanalyse, S. Freud et à son continuateur en France J Lacan. Ce choix est effectivement un parti pris qui en vaut bien d'autres, mais qui se justifie par la capacité de ces deux auteurs à présenter une théorie du sujet, sous la forme de trois instances (moi, surmoi et ça) ou d'une structure (RSI), susceptibles de rendre compte de la division d'un sujet confronté à plusieurs déterminations...et à l'épreuve de la classe.

Enfin, la question de la validation scientifique de tels travaux a été abordée tout au long du texte, sans pouvoir vraiment être développée. Nous la résumerons en précisant que si la généralisation n'a aucun sens ici, les résultats montrent que cette posture permet de produire et de formuler une position du sujet enseignant qui peut être mise en évidence pour un autre cas, sans pour autant prendre la même forme. Elle peut ainsi être un cadre d'analyse des pratiques d'enseignement de l'EPS.

Par exemple, la question du temps «taraude » tous les enseignants, mais elle n'a pas la même influence sur les contenus d'enseignement selon les enseignants, et quelquefois, pour un même enseignant et dans la même classe, pour le traitement de deux APSA différentes. De même, tous les enseignants sont soumis à la « conversion didactique », mais pourtant, chacun retient de sa pratique des éléments de savoir spécifiques à son expérience de pratiquant ou de professeur.

\section{Conclusion.}

Nous retiendrons, en conclusion, plusieurs points qui nous semblent spécifier cette orientation :

- La notion de «conversion didactique » nous permet de rendre compte de l'importance de l'expérience personnelle de l'enseignant dans le traitement didactique d'une APSA. 
- Le désir de transmettre, au-delà de l'intention d'enseigner tel ou tel savoir, existe d'abord, à partir d'une expérience d'un être de sujet.

- La position symbolique de l'enseignant est importante à identifier pour tenter de comprendre, voire d'expliquer, les « gestes » de l'enseignant.

- La confrontation au réel, considéré comme l'impossible à supporter, auquel est soumis tout enseignant, semble être un moyen d'analyse pertinent des pratiques des enseignants d'EPS.

\section{Références}

Andrieux, B. (2007). Le dictionnaire du corps en sciences humaines et sociales. Editions CNRS.

Beillerot, J. (1996). Désir, désir de savoir, désir d’apprendre. In J. Beillerot, C. BlanchardLaville, N. Mosconi (dir.). Pour une clinique du rapport au savoir (pp. 51-75). Paris : L'Harmattan.

Brousse, M. H., \& Labridy, F. (1983). Formation des enseignants d'EPS et psychanalyse, EPS, 184, 31-34.

Blanchard-Laville, C. (2001). Les enseignants, entre plaisir et souffrance. Paris : PUF.

Brousseau, G. (1998). La théorie des situations didactiques. Grenoble: La pensée Sauvage.

Brousseau, G. (1998). Le contrat didactique. Grenoble : La pensée Sauvage.

Buznic-Bourgeacq, P. (2005). L'expérience de l'enseignant et ses implications didactiques : une étude de cas en EPS. In Actes du $5^{\text {ème }}$ colloque international Recherches et formation. IUFM de Nantes.

Caillot, M. (2001). Y a-t-il des élèves en didactique des sciences ? In A. Terrisse (Ed.) La référence au savoir (pp 119-141). Bruxelles : De Boeck Université.

Calmettes, B., Saint Georges M., \& M. Flande, Y. (à paraître en 2008). Analyses de pratiques professionnelles de professeurs de physique stagiaires en situationproblèmes : difficultés repérées, variabilités interindividuelles, In M. F. Carnus, C. Garcia-Debanc \& A. Terrisse (Eds), Analyse des pratiques des enseignants débutants : approche didactique. Grenoble : La pensée Sauvage.

Carnus, M. F. (2001). Analyse didactique du processus décisionnel de l'enseignant d'EPS en gymnastique: une étude de cas croisés. Thèse de doctorat non publiée, Université Paul Sabatier, Toulouse. 381 pages. 
Chemama, R. (1995). Dictionnaire de la psychanalyse. Paris : Larousse.

Chevallard, Y. (1985). La transposition didactique, Grenoble : La pensée Sauvage.

During, B. (1981). La crise des pédagogies corporelles, Editions du Scarabée, Paris: CEMEA.

Freud, S. (1985). Construction dans l'analyse. Résultats, idées problèmes II, Paris : PUF.

Freud, S. (1973). Préface de Freud de Jeunesse à l'abandon, In A Aichhorn (1925) (pp.810). Toulouse : Privat.

Lacan, J. (1975). Séminaire RSI, Ornicar, $n^{\circ} 3$. Paris : Le Seuil.

Laplanche, J., \& Pontalis, J.B. (1971). Vocabulaire de la psychanalyse. Paris : PUF.

Loizon, D. (2004). Analyse des pratiques d'enseignement du judo : identification du savoir transmis a travers les variables didactiques utilisées par les enseignants en club et en EPS, Thèse de doctorat non publiée, Université Paul Sabatier, Toulouse. 352 pages.

Margnes, E. (2002). L'intention didactique dans l'enseignement du judo, des choix culturels d'ordre éthique et technique. Etude de cas de situations didactiques leurs mises en scène- pour des débutants dans la formation initiale en STAPS. Thèse de doctorat non publiée, Université Paul Sabatier, Toulouse. 462 pages.

Levy-Strauss, C. (1962). La pensée sauvage. Paris : Plon.

Perrenoud, P. (1996). Enseigner dans l'urgence, décider dans l'incertitude, Paris : ESF.

Robert, LE (1985). Dictionnaire de la langue française, Paris : Le Robert.

Ria, L., Saury. J., Seve, C., \& Durand, M. (2001). Les dilemmes des enseignants débutants : études lors des premières expériences de classe en EPS, Science et Motricité, 42,47-58.

Salin, M. H. (2002). Les pratiques ostensives d'un enseignant de mathématiques comme objet d'analyse du travail du professeur, In P. Venturini, C. Amade-Escot, \& A. Terrisse (Eds.), Etudes des pratiques effectives: l'approche des didactiques. Grenoble : La pensée sauvage.

Sauvegrain, J. P. (2001). Analyse didactique de la décision de l'élève : étude de cas sur l'utilisation du savoir dans un cycle de lutte en EPS. Thèse de doctorat non publiée, Université Paul Sabatier, Toulouse. 341 pages.

Terrisse, A. (1994). La question de la transmission du savoir dans les APS, Mémoire d'Habilitation à Diriger des Recherches non publié, Toulouse : Université Paul Sabatier, 98 pages. 
Terrisse, A. (2000). Epistémologie de la recherche clinique en sports de combat. In A.

Terrisse (dir.), Recherches en sports de combat et en arts martiaux : état des lieux. (pp. 95-108). Paris : Ed. Revue EPS.

Terrisse, A. (2002). Au-delà du didactique, le clinique, Impulsions, 2, 146-150.

Terrisse, A. (2005). A quoi sert la didactique de l'éducation physique et sportive ?, In Chabchoub, Regards actuels sur les didactiques des disciplines (pp 165-186). Tunis : Publications de l'ATYRED.

Terrisse, A. \& Leziart, Y. (1997). L'émergence d'une notion : la transposition didactique. Entretiens avec Michel Verret (pp. 5-29). Caen: Les Sciences de l'Education vol.30, n³.

Terrisse, A. é Lestel, G. (2005). Le poids de l'expérience dans la didactique de l'EPS, Le cas d'une enseignante "expérimentée ". Premier colloque international des Sciences du Sport de Tunisie. Hammamet : Tunisie.

Verret, M. (1975). Le temps des études. Lille : Librairie Champion.

Van Der Maren, J.M. (1996), Méthode de recherche pour l'éducation. De Boeck Université. Montréal : PUM.

Vigarello, G. (1982), Les vertiges de l'intime. In «Le corps entre illusions et savoirs". Paris : revue Esprit 1982, n². 